\title{
A Comparison between Paclitaxel-Eluting Balloon versus Drug Eluting stent in Denovo Lesions in Coronary Arteries evaluated with MPI
}

H.M.Abo El-Enin, K.A.Shokry and A.M.Ramzy

Cardiology Dept., Faculty of Medicine, Benha Univ.,Benha, Egypt

E-Mail:shaza4545@gmail.com

\begin{abstract}
The use of drug eluting stents (DES) has reduced in-stent restenosis though preventing not only recoil of the vessel wall and late negantive remodeling, but also significantly inhibiting neo-intimal hyperplasia formation. To evaluate the clinical efficacy, outcomes and safety of a drug-eluting balloons for the treatment of de novo coronary artery lesions and To compare the safety and efficacy of the DEB with the outcomes of DES in patients presenting for elective PCI for stable De novo coronary artery lesions. This study included 50 patients whom are being admitted to Kobry El-kobba military hospital for treatment of coronary de novo lesions. Out of the fifty patients included in our study, only three patients (6\%) had positive MPI. Patients with positive MPI had higher age than those with negative MPI [66+-4.5 versus $56.3+-7.7$ years respectively] $(\mathrm{P}=.037)$. In addition, there was a tendency to get positive results with the smaller vessel diameters [mean vessel diameter was 2.50] compared to the wider diameters [mean vessel diameter was $2.64 \pm 0.125$ ] in those with negative but this still not statistically significant $(\mathrm{P}=.054)$. A DEB-only strategy may show comparable short-term results in a mixed clinical patient population with de novo lesions in coronary vessels less than $2.8 \mathrm{~mm}$ in diameter. Despite the higher number of complex patients in our study, we could not prove the superiority of DEB over DES by the use of non-invasive imaging together with the clinical follow up.
\end{abstract}

Keywords: DEB, Eluting, Remodeling, MPI and neo-intimal hyperplasia.

\section{Introduction}

The use of drug eluting stents (DES) majorly reduced in-stent restenosis though preventing not only recoil of the vessel wall and late negative remodeling, but also significantly inhibiting neo-intimal hyperplasia formation. However, concerns about in-stent thrombosis, and the dependency on prolonged dual anti-platelet therapy, and continued restenosis in complex lesion subsets, lead to a search for alternative treatment devices that will tackle restenosis rates without the drawbacks associated with DES. [1]

Drug eluting balloons (DEB) have been developed to overcome the limitations of drug eluting stents (DES), but clinicl results of various DEB studies are still not consistent. The incidence of in-stent restenosis after precutaneous coronary intervention ranges from 5 to $35 \%$ after the implantation of bare metal stent (BMS) and is somehow lower after the implantation of a drug eluting stent in patients who are at moderate risk.(4) Drug-eluting balloons (DEB) are emerging as an effective treatment for in-stent restenosis in both bare-metal stents (BMS) and drug-eluting stents (DES). However, the efficacy of these devices in de novo lesions needs to be established [2].

Small coronary vessels remain a lesion subset in which DES remain associated with relatively high restenosis rates, especially in real-world patients and registries. In some circumstances, the extent of the disease may demand implantation of long stents. Therefore, a suitable alternative to stent implantation to treat small-vessel disease is desirable. In addition, treatment of patients with multivessel disease could benefit from a strategy of DES implantation on the proximal major epicardial vessels and angioplasty with DEB of more distal lesions [3].

Therefore, patients who are at an increased bleeding risk or who are awaiting urgent surgery will mainly receive BMS, where dual antiplatelet therapy is required for one month only. Notably, implantation of BMS is associated with an elevated risk for in-stent restenosis (ISR) of 20 to $30 \%$ compared to 10 to $15 \%$ with DES after one year [4].

In diabetics, representing a high risk population, the relative risk for ISR is further doubled with BMS [5].

Among different DES platforms, several authors have been able to demonstrate an association of (late) stent thrombosis with the polymer and ISR due to a delayed healing and endothelialization process. More recently, bioabsorbable polymers that leave, in effect, a BMS after drug delivery have been developed with promising results. However, all of these proposed DES types require antiplatelet therapy for at least six months [6].

3) DEB deliver higher paclitaxel doses (300 to 600 ug with DEB vs. 100 to 200 ug with DES), and as the drug eluting stent struts commonly cover only $20 \%$ of the injured vessel wall, the larger DEB surface area guarantees more uniform drug delivery [7].

This review will discuss the rationale, concept, and available DEB technologies, along with the preclinical and clinical data available to suPP.ort the DEB as a new technology for endovascular intervention [8].

\section{Subjects and methods}

This is a single center, randomized prospective study that includes 50 patients whom are being admitted to Kobry El-kobba military hospital for treatment of coronary denovo lesions.

\section{Clinical Inclusion criteria}

Age > 18years, Patient providing written informedconsent, Patients with stable angina pectoris (Canadian Cardiovascular Society [CCS] 1, 2 3) or unstable angina pectoris with documented ischemia (CCS 4, Braunwald Class IB-C, IIB-C or IIIB-C), or 
patients with documented silentischemia, Patients who are eligible for coronary revascularization (angioplasty and/orCABG) and Female patients with child bearing potential must have a negative pregnancy test within one week before treatment and must use adequate contraception.

\section{Angiographic Inclusion Criteria:}

Native coronary artery, De novo lesion, Reference vessel diameter $<2.8 \mathrm{~mm}$ by visual estimate, Target lesion with a visually estimated stenosis $>50 \%$, Target lesion length $<28 \mathrm{~mm}$ by visual estimate, A maximum of 2 epicardial vessels requiring revascularization and A maximum of 2 target lesions can be included (in the case of treatment of more than one lesion, the treatment selected will remain the same).

\section{Clinical exclusion criteria}

Patients unable to give informed consent, Patients enrolled in another study with any investigational drug or device within the past 30 days, Patients scheduled for a major surgical intervention within 6 months of enrolment in the study, Patients with acute $(<24 \mathrm{~h})$ or recent ( $\leq 48$ hours) myocardial infarction, Patients with a contraindication to an emergency coronary bypass surgery, Any individual who may refuse a blood transfusion, Patients with serum creatinine $>2.0 \mathrm{mg} / \mathrm{dL}$ or $>180$ umol/L, Patients with severe congestive heart failure, Patients who had a cerebral stroke $<6$ months prior to the index procedure, LVEF (Ejection Fraction) $<30 \%$, Patients with any known allergy, hypersensitivity or intolerance to acetylsalicylic acid (ASA), Clopidogrel, Ticlopidine or Paclitaxel and Any known allergy to contrast medium that cannot be pretreated.

\subsection{Methods}

50 patients were randomized to either PCI (DES) or PTCA (DEB) at Kobry El-Kobba Military Hospital in the period from January 2014 to December 2014. All included patients were followed up by MPI three months after the procedure.

\section{All patients were investigated by:}

1- History taking \& clinical examination:

fulfilling the following data: Age, Gender, Diabetes Miletus, Hypertension, Smoking and Hyperlipidemia

2- 12-lead ECG to recognize rhythm and ischemicchanges.

3- Routinelaboratory: CBC, Blood glucoselevel, Lipidprofile, Renal function and Cardiac enzymes , before and after the procedure \& at any ischemic event through the three months to diagnose periprocedural MI .

4- In-hospital management: All patients will receive low-dose aspirin (ASA), unfractionated heparin (UFH) \& clopidorgel (600 mg loading dose the day before then $75 \mathrm{mg}$ as a maintenance dose for the 3 months of the follow up duration of the study) in addition to conventional treatment (beta-blockers, nitrates, ACEI \&stains)

5- Coronary angiography: Quantitative angiographic measurementswere done to assess:

Number of diseased vessels, -Severity of the coronary lesions, -Presence or absence of calcification, -Diameter of the diseased vessel and -Length of each lesion.

\section{6- Followup}

A- ECG and cardiac enzyme levels were assessed after the procedure. B- Clinical follow-up was performed with visits or telephone contact. Adverse events were monitored throughout the entire study period. C- Myocardial Perfusion Imaging(MPI) was scheduled at 3 months after the procedure for all patients.

\subsection{Statistical analysis}

All data were collected, revised then statistically analyzed using SPSS statistical software version 16.

\section{Results}

The Baseline anatomical criteria are demonstrated in Tables 4. DEB was more used in distal coronary lesions rather that proximal lesions for which DES was more used [ $9(36 \%), 11(44 \%)$ and $5(20 \%)$ patients with DES for distal, mid and proximal vs. 5 (20\%), 4 $(16 \%)$ and $16(64 \%)$ patient with DEB respectively] $(\mathrm{P}<.006)$ (Fig 8, b-c) Fig (1).

DEB was more used in distal coronary lesions rather that proximal lesions for which DES was more used [ $9(36 \%), 11(44 \%)$ and $5(20 \%)$ patients with DEB for distal , mid and proximal vs. 5 (20\%), 4 (16 $\%)$ and $16(64 \%)$ patient with DES respectively] (P < 0.006) Fig 8(c). In the same way, DES were more used in long coronary lesions [mean length was $29.44 \pm 4.673 \mathrm{~mm}]$ while DEB were more used for short lesions [mean length was $24.8 \pm 5.09 \mathrm{~mm}$ ] ( $\mathrm{P}<0.002)$. DES were used for the wider diameters (Mean diameter 2660 micron vs. 2610 microns with DEB group) but this was not statistically significant. (Fig2)

Clinical follow up revealed non eventual course of $46(92 \%)$ patients and non-fatal cardiovascular outcome in the remaining 4 patients $(8 \%)$ patients. Out of those, three patients had chest pain, two of those had positive MPI study while the third on had negative study. Otherwise, No statistical difference between DEB and DES groups for the incidence of complications. Bruce protocol was used in $39(78 \%)$ patients while dipyridamole protocol was used in $11(22 \%)$ patients. Forty-seven (94\%)patients had negative study. Only three $(6 \%)$ patients had positive MPI. Two of them were in DES group, one was symptomatic with chest pain while the other one was asymptomatic. The 3rd positive MPI patient was in the DEB group. No statistical difference was detected between both groups. Fig (3)

The basic demographic, clinical characteristics and presentation categorized upon occurrence of subjective 
symptoms(chest pain) are demonstrated. No statistically significant differences were detected between symptomatic patients with chest pain and asymptomatic ones. Table (1)

The Coronary angiography and MPI results according to subsequent chest pain prevalence are demonstrated. Out of the three patients with chest pain, two of them had positive MPI study, the third one had negative MPI study so the incidence of positive MPI study in positive subjective group was significantly higher that negative group $(\mathrm{P}=.007)$. Table (2)

The basic demographic, clinical characteristics and presentation according to MPI results are demonstrated. Patients with positive MPI had higher age than those with negative MPI [66 \pm 4.5 versus 56.3 \pm 7.7 years respectively] $(\mathrm{P}=.037)$. Otherwise, no other statistically significant difference was detected between both groups. Table (3)

Coronary angiography results according to the MPI follow up outcome are demonstrated. There was a tendency to get positive results in patients whose mean vessel diameters of $2.50 \mathrm{~mm}$ versus $2.64 \mathrm{~mm}$ in those with negative results but not yet statistically significant $(\mathrm{P}=.054)$. Otherwise, no statistically significant difference was detected between both groups. Table (4)

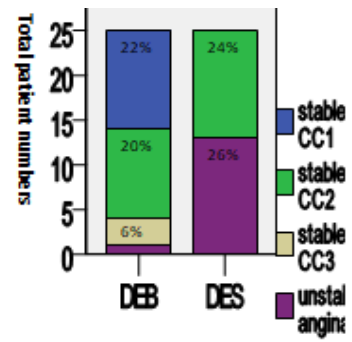

a-Indication of $\mathrm{CA}$

$(\mathrm{P}=<.001)$

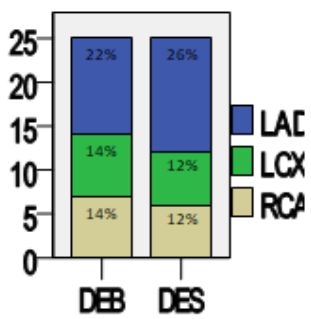

b-Target vessel

$(\mathrm{P}=.852)$

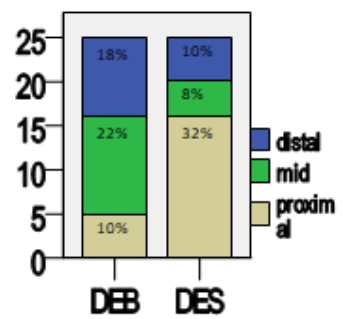

c-Target segment

$(\mathrm{P}=.006)$

Fig (1) (a-c).Indication of intervention and anatomical characteristics in DEB and DES subjected patients

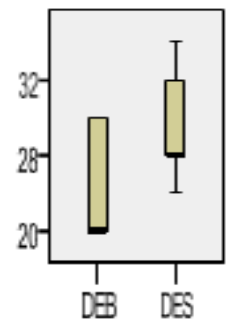

Lesion length

$(\mathrm{P}=.002)$

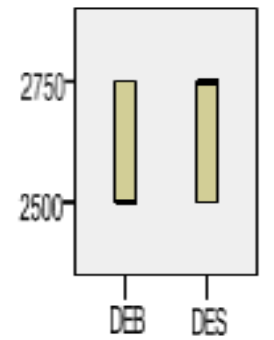

Diameter

$(\mathrm{P}=.162)$

Fig (2)The Intervention details in DEB and DES subjected patients

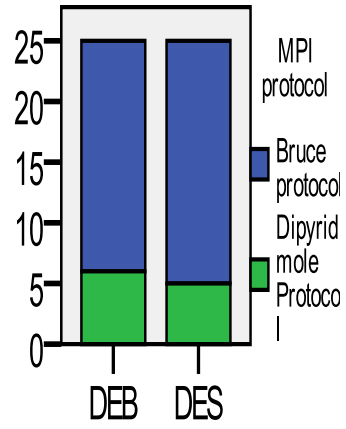

MBI protocol $(\mathrm{P}=.500)$

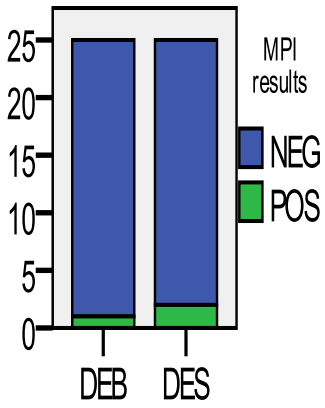

MPI results $(\mathrm{P}=.500)$

Fig (3) The MPI protocol and results in DEB and DES subjected patients 
Table (1) Basic demographic, clinical characteristics and presentation according to subsequent chest pain prevalence.

\begin{tabular}{|c|c|c|c|c|}
\hline & Total No. & $\begin{array}{l}\text { Negative } \\
\text { chest pain } \\
(\text { no=47) }\end{array}$ & $\begin{array}{l}\text { Positive chest pain } \\
(\text { no=3) }\end{array}$ & $\begin{array}{l}P \\
\text { value }\end{array}$ \\
\hline Mean age & & $56.47( \pm 7.8)$ & $63.33( \pm 7.0)$ & .145 \\
\hline Mean BMI & & $29.87( \pm 3.9)$ & $30.67( \pm 2)$ & .733 \\
\hline Male sex & $40(80 \%)$ & $38(95 \%)$ & $2(5 \%)$ & .496 \\
\hline Hypertensive & $28(56 \%)$ & $28(100 \%)$ & $0(0 \%)$ & .079 \\
\hline DM & $47(94 \%)$ & $44(94 \%)$ & $3(6 \%)$ & .827 \\
\hline Smoking & $25(50 \%)$ & $24(96 \%)$ & $1(4 \%)$ & .500 \\
\hline Dyslipidemia & $25(50 \%)$ & $23(92 \%)$ & $2(8 \%)$ & .500 \\
\hline Unstable angina presentation & $14(28 \%)$ & $14(100 \%)$ & $0(0 \%)$ & .114 \\
\hline Pre-procedural ischaemic & $4(8 \%)$ & $3(75 \%)$ & $1(25 \%)$ & \\
\hline ECG changes & & & & .226 \\
\hline
\end{tabular}

$\mathrm{r}_{\mathrm{s}}$ : Spearman coefficient $\quad$ HMGB1: high mobility group box 1 protein $\quad$ BMI: body mass index.

Table (2) Coronary angiography and MPI results according to subsequent chest pain prevalence.

\begin{tabular}{lllll}
\hline & Total No. & $\begin{array}{l}\text { Negative chest pain } \\
(\mathbf{n o = 4 7 )}\end{array}$ & $\begin{array}{l}\text { Positive chest pain } \\
(\mathbf{n o = 3})\end{array}$ & $\begin{array}{l}\text { P } \\
\text { Value }\end{array}$ \\
\hline LAD lesion & $24(48 \%)$ & $23(96 \%)$ & $1(4 \%)$ & .869 \\
LCX lesion & $13(26 \%)$ & $12(92 \%)$ & $1(8 \%)$ & \\
RCA lesion & $13(26 \%)$ & $12(92 \%)$ & $1(8 \%)$ & .484 \\
Distal lesion & $14(28 \%)$ & $13(93 \%)$ & $1(7 \%)$ & \\
Mid lesion & $15(30 \%)$ & $15(100 \%)$ & $0(0 \%)$ & .469 \\
Proximal lesion & $21(42 \%)$ & $19(90 \%)$ & $2(10 \%)$ & .158 \\
Mean DEBDES Diameters & & $2.64( \pm .12)$ & $2.58( \pm .1)$ & .882 \\
Lesion percentage (\%) & & $80.02( \pm 5.5)$ & $85.00( \pm 10)$ & .888 \\
Mean DEBDES Lengths & & $27.15( \pm 5.4)$ & $26.67( \pm 6.1)$ & \\
Lesion length $(\mathbf{m m )}$ & $23.45( \pm 5.3)$ & $23.00( \pm 5.5)$ & $\mathbf{. 0 0 7}$ \\
Pos MPI Results & $3(6 \%)$ & $1(33 \%)$ & $2(67 \%)$ & \\
\hline
\end{tabular}

Table (3) Basic demographic and presentation according to the MPI outcome.

\begin{tabular}{lllll}
\hline & Total No. & Negative MPI (no=47) & Positive MPI (no=3) & P value \\
\hline Mean age & & $56.30( \pm 7.7)$ & $66.00( \pm 4.5)$ & .037 \\
Mean BMI & & $29.87( \pm 3.9)$ & $30.67( \pm 2)$ & .733 \\
Male sex & $40(80 \%)$ & $38(95 \%)$ & $2(5 \%)$ & .496 \\
Hypertensive & $28(56 \%)$ & $27(97 \%)$ & $1(3 \%)$ & .409 \\
DM & $47(94 \%)$ & $44(94 \%)$ & $3(6 \%)$ & .827 \\
Smoking & $25(50 \%)$ & $23(92 \%)$ & $2(8 \%)$ & .500 \\
Dyslipidemia & $25(50 \%)$ & $23(92 \%)$ & $2(8 \%)$ & .500 \\
Unstable angina & $14(28 \%)$ & $13(93 \%)$ & $1(7 \%)$ & .188 \\
presentation & & & & .774 \\
Ischaemic ECG changes & $4(8 \%)$ & $4(100 \%)$ & $0(0 \%)$ & \\
\hline
\end{tabular}

Table (4) Coronary angiography and MPI results according to the MPI follow up outcome.

\begin{tabular}{|c|c|c|c|c|}
\hline & Total No. & $\begin{array}{l}\text { Negative MPI } \\
(n o=47)\end{array}$ & $\begin{array}{l}\text { Positive MPI } \\
(\mathrm{no}=3)\end{array}$ & P Value \\
\hline LAD lesion & $24(48 \%)$ & $23(96 \%)$ & $1(4 \%)$ & .200 \\
\hline LCX lesion & $13(26 \%)$ & $13(100 \%)$ & $0(0 \%)$ & \\
\hline RCA lesion & $13(26 \%)$ & $11(85 \%)$ & $2(15 \%)$ & \\
\hline Distal lesion & $14(28 \%)$ & $13(93 \%)$ & $1(7 \%)$ & .951 \\
\hline Mid lesion & $15(30 \%)$ & $14(93 \%)$ & $1(7 \%)$ & \\
\hline Proximal lesion & $21(42 \%)$ & $20(95 \%)$ & $1(5 \%)$ & \\
\hline Mean DEB\DES Diameters & & $2.64( \pm .1)$ & $2.50( \pm .0)$ & .054 \\
\hline Lesion percentage $(\%)$ & & $82.81( \pm 5.5)$ & $85.33( \pm 5.7)$ & .052 \\
\hline Mean DEB \DES Lengths & & $26.94( \pm 5.1)$ & $30.00( \pm 9.1)$ & .344 \\
\hline Lesion percentage (\%) & & $23.23( \pm 5.07)$ & $26.33( \pm 8.6)$ & .328 \\
\hline
\end{tabular}

\section{Discussion}


DESs are associated with increased bleeding risks caused by the need for prolonged dual antiplatelet therapy (DAPT) and the risk of late and very late stent thrombosis (ST) even though less that first generations of the bare metal stents [9].

DEB use in de novo lesions began by their use for dilatation, then additional BMS deployment. There were significantly different outcomes clinically and angiographically [10].

DES was more used in unstable angina patients [13 (92\%) patients with DES vs. $1(8 \%)$ patient with DEB in unstable angina] rather than chronic stable angina in whom DEB was more used [12(34\%) patients with DES vs. $24(66 \%)$ patients DEB in chronic stable angina] $(\mathrm{P}<0.001)$. Also, DEB was more used in distal coronary lesions rather that proximal lesions for which DES was more used [9(36\%), 11(44\%) and $5(20 \%)$ patients with DEB for distal, mid and proximal vs. 5 $(20 \%), 4(16 \%)$ and $16(64 \%)$ patient with DES respectively] $(\mathrm{P}<.006)$. In the same way, DES were more used in long coronary lesions [mean length was $29.44+-4.673 \mathrm{~mm}$ ] while DEB were more used for shorter lesions [mean length was $24.8+-5.09 \mathrm{~mm}$ ] $(\mathrm{P}<$ .002).

Patients were followed up clinically and with MPI three months after the procedure. Clinical follow up of both groups revealed non eventual course of $46(92 \%)$ patients and non-fatal cardiovascular outcome in the remaining $4(8 \%)$ patients. Out of those, three $(6 \%)$ patients had chest pain, one of those had negative MPI study while the other two patients had positive MPI studies. However, No statistical difference could be detected between DEB and DES groups for the incidence of complications.

Out of the fifty patients included in our study, only three patients $(6 \%)$ had positive MPI. Patients with positive MPI had higher age than those with negative MPI [66+-4.5 versus $56.3+-7.7$ years respectively] $(\mathrm{P}=$ .037). In addition, there was a tendency to get positive results with the smaller vessel diameters [mean vessel diameter was 2.50] compared to the wider diameters[mean vessel diameter was $2.64 \pm 0.125$ ] in those with negative but this still not statistically significant $(\mathrm{P}=.054)$. Despite the increased rate of selection of DEB toward short and distal lesions, DEB alone for de novo coronary lesions could not prove any superior outcome clinically or by nuclear assessment despite the use of DES in more difficult clinical situations as unstable angina or longer lesions.

Most of the studies were trying to assess DEB for In-stent restenosis or combination with BMS. There is some of which studied DEB for de novo lesions in primary PCI settings for acute myocardial infarction. our study excluded both and tried to match both DES and DEB groups for demographic and clinical criteria as age, gender, presence of hypertension or dyslipidemia. The vast majority (94\%) of the ruled in patients were diabetic. However, with the use of DEB for more stable patients; Canadian class 1-2 and or for shorter lesions, we could not prove that DEB had less statistically significant incident of worse outcome by clinical and nuclear follow up. By review of other studies about use of DEB for de novo coronary lesions, we found that their results and conclusions were inconsistent and sometime conflicting. In the following paragraphs, we would try to Fig out the most relevant studies to our study principles.

The Paclitaxel-Eluting PTCA-Balloon Catheter to Treat Small Vessel Coronary Artery Disease (PEPCAD I) study was the first study using a DEB-only strategy in de novo lesions. A DEB was used for dilatation, and the proportion of patients requiring additional BMS deployment was $27 \%$. Despite MACE after 12 months were $15.3 \%$, there was significantly different outcomes clinically and angiographically in favor of the DEBonly strategy, with TLR of $5 \%$ in the DEB-only group and $28 \%$ in the DEB + BMS group [11].

A long-term follow-up registry was performed by Benezet et al., showing persistently low MACE and TLR rates after 36 months and no occurrence of vessel/stent thrombosis. Twenty-five percent received bailout BMS implantation which was not associated with a less favorable outcome (MACE $7.1 \%$ with additional BMS and 9.5\% without BMS) [12].

The PICCOLETO trial compared Dior I DEB versus Taxus Liberté DES in small vessel disease. The study was interrupted after enrollment of two-thirds of patients because of superiority in favor of the DES group. The primary endpoint was percentage diameter stenosis in-segment/in-balloon, and stenosis rates were significantly lower in the DES group; [13].

On the other side, The Balloon Elution and Late Loss Optimization (BELLO) trial was the second randomized study comparing a DEB with DES in small vessel de novo lesions [14], and the IN.PACT Falcon DEB was compared with the Taxus Liberte DES in a sample of 182 patients. The primary endpoint of inballoon (in-stent) LLL was significantly less frequent in DEB compared with DES. The clinical event rate in the DES group was comparable to the rate observed in the DES group from the PICCOLETO study [13]. In contrast, the DEB group in the BELLO study experienced clinical outcomes equal to the extent observed in the DES group. The diverging results from BELLO and PICCOLETO might be due to several factors. While the same DES was used in the two studies, the PICCOLETO study used a first-generation DEB, whereas the BELLO study used a secondgeneration DEB. Predilatation before DEB treatment was done in only $25 \%$ of patients in PICCOLETO vs. $96.8 \%$ in the BELLO study. Predilatation is thought to improve drug uptake by creating microdissections in the vessel wall and thus facilitating drug transport through the intima and media layers [15]. In case of bailout BMS implantation, investigators in the BELLO study were careful with placing the BMS within the DEB treated area, thereby avoiding geographical mismatch, which was not taken into consideration in the PICCOLETO study. The bailout proportion was higher in PICCOLETO than in the BELLO study, and 
this might, especially with the lack of geographical match in PICCOLETO, have contributed to the diverging results. Nonetheless, a subgroup analysis showed, consistent with the findings from PEPCAD I, that bailout implantation in the BELLO study was associated with an increase in LLL of $0.02 \mathrm{~mm}$ in the DEB-only group compared to $0.37 \mathrm{~mm}$ in the $\mathrm{DEB}+\mathrm{BMS}$ group. A recent long-term follow-up of the BELLO study showed that the trend towards superior clinical outcome in the DEB vs. DES group persisted at 24 months [16].

compared the SeQuent Please DEB with POBA. As expected, SeQuent Please DEB was superior to POBA. In accordance with the study by Kleber et al., 9 months angiographic follow-up showed positive remodelling, i.e., negative LLL in 35 out of 49 DEB-treated patients [17].

In our study, Despite the higher number of complex patients, we could not prove the superiority of DEB over DES by the use of non-invasive imaging together with the clinical follow up. Furthermore, newergeneration limus DES (everolimus and zotarolimus) perform even better than sirolimus DES. Thus, by using the best DES on the market, even better DES results would be expected, and the potential benefit of treatment with a DEB might diminish [17].

\section{Conclusion}

A DEB-only strategy may show comparable shortterm results in a mixed clinical patient population with de novo lesions in coronary vessels less than $2.8 \mathrm{~mm}$ in diameter. Despite the higher number of complex patients in our study, we could not prove the superiority of DEB over DES by the use of noninvasive imaging together with the clinical follow up.

\section{References}

[1] JW. Moses, MB. Leon, JJ. Popma, Sirolimuseluting stents versus standard stents in patients with stenosis in a native coronary artery. $\mathrm{N}$ Engl $\mathrm{J}$ Med, Vol. 349, PP.. 1315, 2003.

[2] GW. Stone, SG. Ellis, L. Cannon, Comparison of a polymer-based paclitaxel- eluting stent implantation with a bare metal stent in patients with complex coronary artery disease: a randomized controlled trial.JAMA, Vol. 294, PP.. 1215, 2005.

[3] C. Godino, S. Furuichi, A. Latib, Clinical and angiographicfollow-up of small vessel lesions treated with paclitaxel-eluting stents(from the TRUE Registry). Am J Cardiol, Vol. 102, PP.. 1002, 2008.

[4] C. Stettler, S. Wandel, S. Allemann, Outcomes associated with drug-eluting and bare-metal stents: a collaborative network meta- analysis. Lancet, Vol. 370, PP.. 937, 2007.

[5] U. Stenestrand, SK. James, J. Lindback, Safety and efficacy of drug-eluting vs. bare metal stents in patients with diabetes mellitus: long-term followup in the Swedish Coronary Angiography and
Angioplasty Registry (SCAAR). Eur Heart J, Vol. 31, PP.. 177, 2010.

[6] F. Van Buuren, JB. Dahm, D. Horskotte, Stent restenosis and thrombosis: etiology, treatment, and outcomes. Minerva Med, Vol. 103, PP.. 503-511, 2012.

[7] L. King, R. Byrne, J. Mehilli, Five-year clinical outcomes of a polymer-free sirolimus-eluting stent versus a permanent polymer paclitaxel-eluting stent: Final results of the intracoronary stenting and angiographic restenosis-Test equivalence between two drug- eluting stents (ISAR-TEST) trial. Catheter. Cardiovasc. Interv, Vol. 81, PP.. 23, 2013.

[8] B. Cortese, A. Bertoletti, Paclitaxel coated balloons for coronary artery interventions: a comprehensive review of preclinical and clinical data. Int $\mathrm{J}$ Cardiol; Vol. 161, PP.. 4, 2012.

[9] M. Chang, DW. Park, Optimal duration of dual antiplatelet therapy after implantation of drugeluting stents: shorter or longer? Cardiol Ther, Vol. 3, PP.. 1, 2014.

[10] J. Wöhrle, M. Zadura, S. Möbius-Winkler, SeQuentPlease World Wide Registry: clinical results of SeQuent please paclitaxel-coated balloon angioplasty in a large-scale, prospective registry study. J Am Coll Cardiol; Vol. 60, PP.. 1733, 2012.

[11] M. Unverdorben, FX. Kleber, H. Heuer, Treatment of small coronary arteries with a paclitaxel-coated balloon catheter. Clin Res Cardiol; Vol. 99, PP.. 165, 2010.

[12] J. Benezet, A. Gutiérrez-Barrios, A. Agarrado, Paclitaxel-coated balloon angioplasty for de novo coronary lesions: a long-term follow-up study. Minerva Cardioangiol, Vol. 64, PP.. 15, 2016.

[13] B. Cortese, A. Micheli, A. Picchi, Paclitaxelcoated balloon versus drug-eluting stent during PCI of small coronary vessels, a prospective randomised clinical trial. The PICCOLETO study. Heart; Vol. 96, PP.. 1291, 2010.

[14] A. Latib, A. Colombo, F. Castriota, A randomized multicenter study comparing a paclitaxel drug-eluting balloon with a paclitaxeleluting stent in small coronary vessels: the BELLO (balloon elution and late loss optimization) study. J Am Coll Cardiol, Vol. 60, PP.. 2473, 2012.

[15] A. Belkacemi, P. Agostoni, HM. Nathoe, First results of the DEB-AMI (drug eluting balloon in acute ST-segment elevation myocardial infarction) trial: a multicenter randomized comparison of drug-eluting balloon plus bare-metal stent versus bare-metal stent versus drug-eluting stent in primary primary percutaneous coronary intervention with 6-month angiographic, intravascular, functional, and clinical outcomes. J Am Coll Cardiol,Vol. 59, PP.. 2327, 2012.

[16] T. Naganuma, A. Latib, GA. Sgueglia, A 2-year follow-up of a randomized multicenter study 
comparing a paclitaxel drug-eluting balloon with a paclitaxel-eluting stent in small coronary vessels the BELLO study. Int J Cardiol,Vol. 184, PP.. 17, 2015.

[17] AY. Her, SH. Ann, GB. Singh, Comparison of paclitaxel-coated balloon treatment and plain old balloon angioplasty for de novo coronary lesions. Yonsei Med J Yonsei Univ Coll Med, Vol. 57, PP..337, 2016.
[18] R. Piccolo, GG. Stefanini, A. Franzone, Safety and efficacy of resolute zotarolimus-eluting stents compared with everolimus-eluting stents: a metaanalysis. Circ Cardiovasc Interv, Vol. 8, PP. 002223, 2015. 